\title{
Insulinosensibilisateurs (metformine/glitazones) : niveau de preuve et controverse
}

\author{
Insulin sensitizers (metformin/glitazones): \\ Level of evidence and controversy
}

\author{
A.-J. Scheen \\ Service de diabétologie, \\ nutrition et maladies métaboliques, \\ Unité de pharmacologie clinique, \\ Département de médecine, \\ C.H.U. Sart Tilman, Liège, Belgique. \\ Président de la Société francophone \\ du diabète.
}

\begin{abstract}
Résumé
Au vu du rôle délétère de l'insulinorésistance, un médicament insulino-sensibilisateur devrait normalement réduire l'incidence des complications cardiovasculaires du diabète de type 2. Dans l'étude UKPDS, la metformine a réduit le risque d'infarctus du myocarde et la mortalité cardiovasculaire chez des ptients récemment diagnostiqués et à faible risque, résultats qui mériteraient d'être confirmés chez des patients à haut risque cardiovasculaire. Les glitazones (des agents insulinosensibilisateurs plus spécifiques) avaient suscité beaucoup d'espoirs. Cependant la rosiglitazone est tombée de son piédestal après la suspicion d'une augmentation du risque coronarien (non réellement prouvé). Dans PROactive, conduite chez des patients à haut risque cardiovasculaire, la pioglitazone a donné des résultats discutables : la signification statistique n'était pas atteinte pour le principal critère d'évaluation composite étendu, mais bien pour le critère secondaire principal restreint pré-spécifié ou dans des analyses post-hoc. Ainsi, si les données disponibles apparaissent plutôt favorables concernant les insulino-sensibilisateurs, les preuves sont fragiles, d'où la controverse récurrente.
\end{abstract}

Mots-clés : Diabète de type 2 - macroangiopathie - maladies cardiovasculaires metformine - pioglitazone - rosiglitazone.

\begin{abstract}
Summary
Because of the deleterious role attributed to insulin resistance, insulin sensitizing agents should theoretically reduce the incidence of cardiovascular complications in type 2 diabetes. In the UKPDS, metformin reduced the risk of myocardial infarction and cardiovascular mortality in a rather small group of recently diagnosed patients at a low cardiovascular risk. These results deserve confirmation in a larger study with patients a high cardiovascular risk. Glitazones, as more specific insulin sensitizers, raised much hope. However, rosiglitazone fell from its pedestal after the suspicion of an increased risk of myocardial infarction. In PROactive among patients at high cardiovascular risk, pioglitazone gave positive but questionable results: statistical significance was not reached regarding the large composite primary endpoint, but well regarding the prespecified more focused principal secondary endpoint or in various post-hoc analyses. Thus, even if data with insulin sensitizers appear globally rather promising, evidence is rather weak, therefore leading to recurrent controversy.
\end{abstract}

Key-words: Cardiovascular disease - macroangiopathy - metformin pioglitazone - rosiglitazone - type 2 diabetes.

\section{Correspondance \\ André J. Scheen \\ nutrition et maladies métaboliques \\ Unité de pharmacologie clinique \\ Département de médecine \\ C.H.U. Sart Tilman \\ B-4000 Liège - Belgique \\ andre.scheen@chu.ulg.ac.be}




\section{Introduction}

- Le diabète de type 2 (DT2) est une maladie complexe qui se caractérise par deux anomalies principales (souvent associées, en proportion variable, par ailleurs), à savoir un déficit de l'insulinosécrétion, et une insulinorésistance [1]. Suite aux travaux de quelques grands leaders d'opinion américains, comme Reaven [2] et DeFronzo [3], le rôle de l'insulinorésistance a été mis en exergue, non seulement dans la physiopathologie du DT2, mais aussi dans celle des maladies cardiovasculaires (CV), en particulier l'insuffisance coronaire, avec l'émergence du concept de syndrome (cardio-)métabolique. Aussi, les médicaments susceptibles d'améliorer l'action de l'insuline (insulino-sensibilisateurs) ont-ils été proposés pour traiter l'hyperglycémie du DT2, avec l'espoir de réduire également le risque de complications CV associées à cette maladie [4].

- Les premiers éléments de preuve ont été apportés, en 1998, avec les résultats positifs du bras metformine dans l'étude UKPDS [5], même si la metformine ne peut pas être considérée comme un médicament insulino-sensibilisateur stricto sensu [6]. Peu après ont été commercialisées les thiazolidinediones (glitazones), médicaments ayant une action plus spécifique et plus puissante sur l'insulinorésistance, et sur lesquels de grands espoirs avaient été placés [7]. Finalement, c'est la déception qui semble l'avoir emporté après la publication des résultats, certes positifs, mais controversés, de l'étude PROactive avec la pioglitazone [8] et, surtout, la véritable bombe lancée par Nissen et Wolski, en 2007, lorsqu'ils ont publié les résultats d'une méta-analyse suggérant une augmentation, et non une diminution, des infarctus du myocarde (IDM) avec la rosiglitazone, associée à une tendance à l'augmentation de la mortalité CV [9]. La controverse a été réactivée, en 2014, avec la publication des résultats négatifs avec un agoniste mixte des peroxisome proliferator-activated receptors (PPAR) PPAR $\gamma / P P A R \alpha$, l'aléglitazar, chez des patients DT2 ayant présenté un accident coronarien aigu récent [10].
- Dans leur version, actualisée début 2015, de la prise de position pour la prise en charge de l'hyperglycémie dans le DT2, l'American Diabetes Association (ADA) et l'European Association for the Study of Diabetes (EASD) ont placé la metformine en première intention, et les glitazones en seconde ou troisième intention (sur le même plan que d'autres approches anti-hyperglycémiantes). II faut cependant bien reconnaître que la problématique CV n'était guère mise en avant dans les critères de choix proposés, plutôt centrés sur le risque hypoglycémique, la prise de poids, et le coût du traitement [11].

- Le but de cet article de revue est de résumer les principaux résultats des essais cliniques randomisés, des études observationnelles et des méta-analyses consacrés à la metformine, d'une part, aux thiazolidinediones (pioglitazone, rosiglitazone), d'autre part, en centrant notre attention sur les événements CV comme critères forts, et non sur de simples critères intermédiaires comme des facteurs/marqueurs de risque. Dans la discussion finale, nous envisagerons également les controverses soulevées par le rôle de ces molécules dans la protection CV qu'elles sont susceptibles d'apporter à la population DT2. II faut noter que les effets, tant de la metformine que des glitazones, sur les lésions de microangiopathie ont été moins bien évalués et, au final, n'apparaissent pas très évidents (mise à part, peut-être, une réduction de la microalbuminurie avec les glitazones) ; ils ne seront pas abordés spécifiquement dans cet article, par souci de concision.

\section{Résultats des études interventionnelles et observationnelles}

\section{Metformine}

\section{Essais cliniques contrôlés}

- La metformine a connu un succès grandissant après la publication des résultats de I'UKPDS, en 1998 [5]. Dans cette étude, le sous-groupe de patients obèses traités par metformine a présenté une réduction significative des événements $C V$ et de la mortalité (tableau I), alors que semblable effet favorable n'avait pu être démontré dans le groupe principal ayant bénéficié du traitement intensif à base d'insuline ou de sulfamides hypoglycémiants. Comme l'amélioration du contrôle glycémique était comparable avec les deux approches pharmacologiques, l'effet favorable de la metformine sur les complications CV a été attribué aux effets propres de la molécule, même si ceux-ci restent, à vrai dire, assez mystérieux. Ces données positives ont été confirmées lors d'un suivi à 10 ans [12]. La protection a concerné les décès liés au diabète, les décès de toutes causes, les IDM, mais pas les accidents vasculaires cérébraux (AVC), ni les complications liées à une artériopathie périphérique (tableau I). Cependant, l'existence de cet effet de protection CV de la metformine a été contestée, essentiellement pour des raisons méthodologiques liées à l'étude UKPDS [13] (voir plus loin discussion).

Tableau I. Comparaison des résultats de l'intensification du contrôle glycémique avec la metformine à la fin de I'UKPDS [5] et à la fin de la période d'observation supplémentaire de 10 années [12] dans le groupe de patients avec surcharge pondérale. Les résultats sont exprimés par les hazard ratio (HR) [avec intervalles de confiance à $95 \%$ ] par rapport au groupe diabétique témoin.

\begin{tabular}{|c|c|c|}
\hline & Fin d'étude UKPDS [5] & Fin du suivi à 10 ans [12] \\
\hline Tout événement lié au diabète & $0,68[0,53-0,87] p=0,003$ & $0,79[0,66-0,95] p=0,01$ \\
\hline $\begin{array}{l}\text { Complications } \\
\text { de microangiopathie }\end{array}$ & $0,71[0,43-1,19] p=0,19$ & $0,84[0,60-1,17] p=0,31$ \\
\hline Décès liés au diabète & $0,58[0,37-0,91] p=0,017$ & $0,70[0,53-0,92] p=0,01$ \\
\hline Décès de toutes causes & $0,73[0,59-0,89] p=0,002$ & $0,64[0,45-0,91] p=0,011$ \\
\hline Infarctus du myocarde & $0,61[0,41-0,89] p=0,010$ & $0,67[0,51-0,89] p=0,005$ \\
\hline Accident vasculaire cérébral & $0,59[0,29-1,18] p=0,13$ & $0,80[0,50-1,27] p=0,35$ \\
\hline Artériopathie périphérique & $0,74[0,26-2,09] p=0,57$ & $0,63[0,32-1,27] p=0,19$ \\
\hline
\end{tabular}


- L'étude COSMIC est une étude randomisée ouverte d'une année, en groupes parallèles, dans laquelle des patients DT2 imparfaitement contrôlés par régime ou par sulfamides hypoglycémiants ont reçu, soit de la metformine $(n=7227)$, soit un autre traitement antidiabétique usuel ( $n=1505)$ [14]. Le critère d'évaluation composite, comprenant les événements indésirables sévères, les hospitalisations et les décès, a été observé de façon comparable dans les deux groupes $(10,3 \%$ [intervalle de confiance à $95 \%$, IC $95 \%: 9,6-11,1 \%]$ versus $11,0 \%$ [IC $95 \%: 9,5-12,7 \%]$, respectivement ; $p=0,431$ ). Les événements CV étaient la principale cause de décès, sans différence entre les deux groupes $(0,7 \%$ pour la metformine, et $0,9 \%$ pour le traitement comparateur), que ce soit les événements cardiaques $(0,6$ versus $0,8 \%$, respectivement; IDM : 0,3 versus $0,3 \%$, arrêt cardiorespiratoire: 0,1 versus $0,0 \%$, insuffisance cardiaque : 0,1 versus $0,3 \%$ ) ou vasculaires $(0,1$ versus $0,3 \%$, respectivement).

- Dans un essai clinique multicentrique, mais avec un échantillon limité, des patients DT2 traités par insuline ont reçu, soit la metformine $(850 \mathrm{mg}, 1$ à 3 fois par jour ; $n=196$ ), soit un placebo $(n=190)$ et ont été suivis pendant 4,3 années. La metformine n'a pas été associée à une amélioration du critère composite d'évaluation primaire qui comprenait des complications microvasculaires et macrovasculaires et la mortalité. Par contre, dans le groupe metformine, l'incidence des événements macrovasculaires (définie au départ comme critère secondaire spécifique) a été significativement plus basse que dans le groupe placebo (hazard ratio, $\mathrm{HR}=0,61[\mathrm{IC} 95 \%=0,40-0,94]$; $p=0,02)$. Le nombre de sujets à traiter pour prévenir un événement $C V$ était de 16,1 [IC $95 \%$ : 9,2-66,6] [15].

- Dans l'étude DIGAMI 2, réalisée chez des patients DT2 dans les suites immédiates d'un syndrome coronarien aigu, le groupe traité par metformine a eu un pronostic plus favorable que le groupe traité par sulfamides hypoglycémiants ou par insuline, avec une réduction significative de la mortalité après un suivi médian de 4,1 années ( $H R=0,65$
[IC $95 \%: 0,47-0,90] ; p=0,0094$ ) [16]. Cependant, la différence en termes de décès était plus marquée pour les décès secondaires à un cancer $(\mathrm{HR}=0,25$ [IC $95 \%: 0,08-0,83] ; p=0,0235)$ que pour ceux d'origine CV (HR= 0,72 [IC $95 \%$ : $0,49-1,06] ; p=0,0995)$ [16].

\section{Études observationnelles de cohorte}

Plusieurs études observationnelles de cohorte, réalisées dans divers pays, ont analysé l'incidence des événements $\mathrm{CV}$, en ce compris la mortalité, chez des patients DT2 traités par différents médicaments antidiabétiques. Après ajustement pour divers facteurs potentiellement confondants, la metformine s'est révélée être associée à moins d'événements CV que les sulfamides hypoglycémiants [17-20]. De même, la metformine s'est montrée plus favorable que l'acarbose, l'inhibiteur des $\alpha$-glucosidases de référence [21], et également légèrement meilleure, quoi que de façon non significative, que la sitagliptine, le leader de la classe des inhibiteurs de la dipeptidylpeptidase-IV (DPP-4) [22]. Dans le registre REACH, la prise de metformine a été associée à une réduction de la mortalité totale $(\mathrm{HR}=0,76$ [IC $95 \%: 0,65-0,89]$; $p<0,001)$, de la mortalité CV $(\mathrm{HR}=0,79$ [IC $95 \%: 0,65-0,96] ; p=0,002$ ) et d'un critère composite comprenant les décès, les IDM et les AVC (HR = 0,88 [IC $95 \%: 0,79-0,99] ; p=0,04)$ [23]. Cette étude, et d'autres, suggèrent que la metformine peut s'avérer utile pour réduire le risque CV, y compris dans des sousgroupes de patients chez lesquels elle était classiquement considérée comme contre-indiquée [24].

\section{Méta-analyses}

- Plusieurs méta-analyses ont rassemblé les résultats des essais cliniques qui ont rapporté la fréquence des événements $C V$ sous metformine, en comparaison avec un autre médicament antidiabétique ou un placebo. Les résultats varient selon les méta-analyses considérées, avec une réduction de la mortalité $\mathrm{CV}$ dans une première métaanalyse publiée [25], mais pas vraiment confirmée dans deux autres [13, 26] (tableau II). II est cependant important que noter que la plupart des essais inclus dans ces méta-analyses n'étaient pas dédiés à la problématique $\mathrm{CV}$ et que, pour beaucoup d'entre eux, la durée de suivi était limitée et le nombre d'événements relativement faible. Les deux méta-analyses les plus récentes rapportent une réduction de $10 \%$ des IDM, non significative cependant, alors qu'il n'y a pas de différence en ce qui concerne la mortalité CV, la mortalité totale ou le risque d'insuffisance cardiaque (tableau II) $[13,26]$. Une métaanalyse s'est intéressée uniquement aux essais ayant enrôlé des patients avec un DT2 insulino-traité, ce qui limite encore davantage le nombre d'essais, et donc le nombre d'événements [27]. Ce travail n'a pas rapporté de différences significatives entre les sujets traités par metformine versus ceux non traités par metformine, que ce soit pour la mortalité CV (odds ratio, OR = 1,70 [IC $95 \%$ : $0,35-8,30])$ ou un composite des événements macrovasculaires $(\mathrm{OR}=0,98$ [IC $95 \%: 0,79-1,22]$ ).

\section{Pioglitazone}

\section{Essais cliniques contrôlés}

- PROactive est une étude prospective, contrôlée versus placebo, réalisée chez 5238 patients avec un DT2 et une maladie macrovasculaire connue [8]. Les patients ont reçu, de façon aléatoire, soit de la pioglitazone (titration forcée de 15 à $45 \mathrm{mg} /$ jour), soit un placebo, en association à leur traitement hypoglycémiant habituel et à leurs autres médicaments. Après un suivi moyen de 34,5 mois, le critère d'évaluation primaire - vaste critère composite comprenant la mortalité toutes causes, I'IDM non fatal (y compris I'IDM silencieux), I'AVC non fatal, le syndrome coronaire aigu, les procédures de revascularisation endovasculaire ou chirurgicale au niveau des artères coronaires ou des artères des membres inférieurs, et l'amputation au-dessus de la cheville - n'a pas été significativement modifié (HR = 0,90 [IC $95 \%: 0,80-1,02]$; $p=0,095)$. En revanche, le principal critère d'évaluation secondaire, plus restreint et comprenant la mortalité toutes causes, I'IDM non mortel et l'AVC non mortel (soit un critère composite se rapprochant de celui classiquement utilisé dans les 
grandes études de prévention CV), a été significativement diminué dans le groupe pioglitazone par rapport au groupe placebo (HR = 0,84 [IC $95 \%: 0,72-0,98]$; $p=0,027$ ) (tableau III). Comme attendu, il y a eu davantage de cas d'insuffisance cardiaque dans le groupe pioglitazone que dans le groupe placebo: tout cas d'insuffisance cardiaque (11\% versus $6 \% ; p<0,0001)$, et cas nécessitant une hospitalisation (6\% versus $4 \%$; $p=0,007$ ) [8].

- Diverses analyses post-hoc de l'étude PROactive ont été réalisées ; leurs principaux résultats ont été résumés dans un article précédent [28]. Globalement, elles sont toutes favorables et démontrent dans le groupe pioglitazone par rapport au groupe placebo, notamment, une réduction significative des récidives d'IDM chez les patients avec antécédents coronariens (HR = 0,72 [IC $95 \%$ : 0,52-0,99]; $p=0,045$ ) [29] et, davantage encore, des récidives d'AVC chez les patients avec antécédents cérébro-vasculaires (HR = 0,53 [IC $95 \%$ : 0,34-0,85]; $p=0,0085$ ) (tableau III) [30].

\section{Études observationnelles de cohorte}

Quelques études observationnelles de cohorte ont comparé le pronostic CV des patients DT2 traités par différentes médications antidiabétiques. Dans ces études, la pioglitazone s'est révélée être associée à un meilleur pronostic CV (hormis le risque d'insuffisance cardiaque qui était accru) que la metformine [18] ou qu'un sulfamide hypoglycémiant chez des patients par ailleurs déjà traités par metformine [31]. De plus, quelques études observationnelles ont comparé le devenir de patients DT2 traités par glitazones, et ont montré que la pioglitazone était associée à un meilleur pronostic que la rosiglitazone [18, 32].

\section{Méta-analyses}

Plusieurs méta-analyses ont étudié l'influence de la pioglitazone sur le risque d'événements CV (tableau II) [33, 34]. À nouveau, à l'exclusion de l'étude PROactive, aucun des essais cliniques inclus dans ces méta-analyses n'avait été conçu pour étudier spécifiquement les événements CV ; de plus, ils étaient de relativement
Tableau II. Principales méta-analyses publiées concernant les effets de la metformine, de la pioglitazone et de la rosiglitazone sur les événements cardiovasculaires (CV) et la mortalité. Les résultats sont exprimés par l'odds ratio (OR) [avec l'intervalle de confiance à $95 \%$ ] des événements sous médicament insulino-sensibilisateur versus le comparateur (placebo ou autre antidiabétique).

\begin{tabular}{|c|c|c|c|c|}
\hline & $\begin{array}{c}\text { Infarctus } \\
\text { du myocarde }\end{array}$ & Mortalité CV & Mortalité totale & $\begin{array}{c}\text { Insuffisance } \\
\text { cardiaque }\end{array}$ \\
\hline \multicolumn{5}{|l|}{ Metformine } \\
\hline $\begin{array}{l}\text { Selvin et al, } \\
2008 \text { [25] }\end{array}$ & $\begin{array}{c}0,85 \\
{[0,69-1,05](b)}\end{array}$ & $\begin{array}{c}0,74 \\
{[0,62-0,89]}\end{array}$ & $\begin{array}{c}0,81 \\
{[0,60-1,08]}\end{array}$ & ND \\
\hline $\begin{array}{l}\text { Lamanna et al, } \\
2011 \text { [26] }\end{array}$ & $\begin{array}{c}0,90 \\
{[0,71-1,14]}\end{array}$ & $\begin{array}{c}0,923(c) \\
{[0,361-2,320]}\end{array}$ & $\begin{array}{c}1,103(d) \\
{[0,804-1,513]}\end{array}$ & $\begin{array}{c}1,12 \\
{[0,25-9,04]}\end{array}$ \\
\hline $\begin{array}{l}\text { Boussageon } \\
\text { et al, } 2012 \text { [13] }\end{array}$ & $\begin{array}{c}0,90 \\
{[0,74-1,09]}\end{array}$ & $\begin{array}{c}1,05 \\
{[0,67-1,64]}\end{array}$ & $\begin{array}{c}0,99 \\
{[0,75-1,31]}\end{array}$ & $\begin{array}{c}1,03 \\
{[0,67-1,59]}\end{array}$ \\
\hline \multicolumn{5}{|l|}{ Pioglitazone } \\
\hline $\begin{array}{l}\text { Lincoff et al, } \\
2007 \text { [33] }\end{array}$ & $\begin{array}{c}0,81 \\
{[0,64-1,02]}\end{array}$ & $\begin{array}{c}0,82 \\
{[0,72-1,11](e)}\end{array}$ & $\begin{array}{c}0,92 \\
{[0,76-1,11]}\end{array}$ & $\begin{array}{c}1,41 \\
{[1,14-1,76]}\end{array}$ \\
\hline $\begin{array}{l}\text { Mannucci et al, } \\
2008 \text { [34] (a) }\end{array}$ & $\begin{array}{c}0,82 \\
{[0,55-1,23]}\end{array}$ & $\begin{array}{c}0,49 \\
{[0,21-1,15]}\end{array}$ & $\begin{array}{c}0,41 \\
{[0,23-0,72]}\end{array}$ & $\begin{array}{c}1,32 \\
{[0,88-1,98]}\end{array}$ \\
\hline \multicolumn{5}{|l|}{ Rosiglitazone } \\
\hline $\begin{array}{l}\text { Nissen \& Wolski, } \\
2007 \text { [9] }\end{array}$ & $\begin{array}{c}1,43 \\
{[1,03-1,98]}\end{array}$ & $\begin{array}{c}1,64 \\
{[0,98-2,74]}\end{array}$ & $\begin{array}{c}1,18 \\
{[0,89-1,55]}\end{array}$ & ND \\
\hline $\begin{array}{l}\text { Nissen \& Wolski, } \\
2010 \text { [43] }\end{array}$ & $\begin{array}{c}1,28 \\
{[1,02-1,63]}\end{array}$ & $\begin{array}{c}1,03 \\
{[0,78-1,36]}\end{array}$ & $\begin{array}{c}0,98 \\
{[0,82-1,17]}\end{array}$ & ND \\
\hline $\begin{array}{l}\text { Mannucci et al, } \\
2010[44]\end{array}$ & $\begin{array}{c}1,14 \\
{[0,90-1,45]}\end{array}$ & $\begin{array}{c}0,94 \\
{[0,68-1,29]}\end{array}$ & $\begin{array}{c}0,93 \\
{[0,76-1,14]}\end{array}$ & $\begin{array}{c}1,69 \\
{[1,21-2,36]}\end{array}$ \\
\hline
\end{tabular}

(a) Données dans les essais avec la pioglitazone, mais sans inclure PROactive.

(b) Pas de données limitées aux infarctus du myocarde, données rapportées correspondent à la morbidité CV. (c) $\mathrm{OR}=0,554$ [IC $95 \%: 0,356-0,890]$ dans les essais avec la metformine en monothérapie.

(d) Diminution avec metformine en monothérapie, mais augmentation avec metformine ajoutée à un sulfamide hypoglycémiant.

(e) Mortalité CV non disponible : résultats exprimés par critère composite (mortalité + infarctus du myocarde + accident vasculaire cérébral)

CV : cardiovasculaire ; ND : non disponible.

\section{Les points essentiels}

- Dans l'étude UKPDS, la metformine réduit les événements cardiovasculaires et la mortalité, à la fin de l'essai et 10 ans plus tard.

- Les effets macrovasculaires positifs de la metformine devrait être confirmés dans une grande étude prospective chez des patients diabétiques de type 2 à haut risque cardiovasculaire.

- La rosiglitazone a été très décriée après une méta-analyse controversée de 2007 et retirée du marché dans de nombreux pays. Pourtant, les données actualisées semblent moins défavorables, si bien qu'aux États-Unis, la Food and Drug Administration (FDA) a fait quelque peu marche arrière récemment.

- Quoi qu'il en soit, le profil de la pioglitazone paraît plus favorable que celui de la rosiglitazone, à la fois sur le profil lipidique et sur la survenue d'événements cardiovasculaires, dans les études épidémiologiques disponibles.

- Dans l'étude PROactive, la pioglitazone réduit significativement le critère composite (mortalité, infarctus du myocarde, accidents vasculaires cérébraux), ainsi que la récidive des accidents coronariens ou cérébro-vasculaires, mais pas le critère composite élargi initialement choisi comme critère de jugement principal par les investigateurs. - Ce critère composite élargi a été très critiqué en raison de sa grande hétérogénéité, incluant également des événements touchant les membres inférieurs, mais aussi combinant des événements « maladie-dépendants » (infarctus du myocarde, par exemple) et « investigateur-dépendants » (procédures de revascularisation, par exemple).

- Il y a peu de données concernant un éventuel rôle protecteur de la metformine ou des glitazones vis-à-vis des complications de microangiopathie. 
Tableau III. Critères d'évaluation cardiovasculaire dans divers sous-groupes de PROactive. Les résultats correspondent aux valeurs de hazard ratio (HR) [avec l'intervalle de confiance à $95 \%$ ] chez les patients traités par pioglitazone versus ceux recevant le placebo.

\begin{tabular}{|c|c|c|c|c|c|c|}
\hline $\begin{array}{c}\text { Groupes } \\
\text { de patients }\end{array}$ & Référence & $\begin{array}{c}\mathbf{n} \\
\text { pio-glitazone } \\
\text { versus } \\
\text { placebo }\end{array}$ & $\begin{array}{c}\text { Critère } \\
\text { composite } \\
\text { primaire (a) }\end{array}$ & $\begin{array}{c}\text { Critère } \\
\text { secondaire } \\
\text { principal (b) }\end{array}$ & $\begin{array}{c}\text { Mortalité } \\
\text { de toutes causes }\end{array}$ & $\begin{array}{c}\text { Critère } \\
\text { d'évaluation } \\
\text { spécifique }\end{array}$ \\
\hline $\begin{array}{l}\text { Ensemble } \\
\text { des patients }\end{array}$ & $\begin{array}{l}\text { Dormandy et al, } \\
2005 \text { [8] }\end{array}$ & 2605 vs 2633 & $\begin{array}{l}H R=0,90 \\
{[0,80-1,02]} \\
p=0,095\end{array}$ & $\begin{array}{l}H R=0,84 \\
{[0,72-0,98]} \\
p=0,027\end{array}$ & $\begin{array}{l}\mathrm{HR}=0,96 \\
{[0,78-1,18]} \\
p=0,68\end{array}$ & $\begin{array}{l}\mathrm{HR}=0,82 \\
{[0,70-0,97]} \\
p=0,020 \text { (c) }\end{array}$ \\
\hline $\begin{array}{l}\text { Patients avec } \\
\text { antécédents d'IDM }\end{array}$ & $\begin{array}{l}\text { Erdmann et al, } \\
2007 \text { [29] }\end{array}$ & 1230 vs 1215 & $\begin{array}{l}\mathrm{HR}=0,88 \\
{[0,75-1,04]} \\
p=0,135\end{array}$ & $\begin{array}{l}\mathrm{HR}=0,81 \\
{[0,65-1,01]} \\
p=0,058\end{array}$ & $\begin{array}{l}\mathrm{HR}=0,85 \\
{[0,63-1,15]} \\
p=0,2873\end{array}$ & $\begin{array}{l}\mathrm{HR}=0,72 \\
{[0,52-0,99]} \\
p=0,045(\mathrm{~d})\end{array}$ \\
\hline $\begin{array}{l}\text { Patients avec } \\
\text { antécédents d'AVC }\end{array}$ & $\begin{array}{l}\text { Wilcox et al, } \\
2007 \text { [30] }\end{array}$ & 486 vs 498 & $\begin{array}{l}\mathrm{HR}=0,78 \\
{[0,60-1,02]} \\
p=0,067\end{array}$ & $\begin{array}{l}\mathrm{HR}=0,78 \\
{[0,58-1,06]} \\
p=0,110\end{array}$ & $\begin{array}{l}\mathrm{HR}=0,96 \\
{[0,64-1,44]} \\
p=0,843\end{array}$ & $\begin{array}{l}\mathrm{HR}=0,53 \\
{[0,34-0,85]} \\
p=0,0085 \text { (e) }\end{array}$ \\
\hline
\end{tabular}

IDM : infarctus du myocarde ; AVC : accident vasculaire cérébral.

(a) Mortalité de toute cause, IDM non mortels, AVC non mortels, syndromes coronariens aigus, procédures de revascularisation coronaire et périphérique, amputations au-dessus de la cheville.

(b) Mortalité de toute cause, IDM non mortels, AVC non mortels.

(c) Décès cardiovasculaire, IDM non mortels, AVC non mortels.

(d) IDM mortels et non mortels (excluant les IDM silencieux).

(e) AVC mortels et non mortels.

Tableau IV. Comparaison des critères d'évaluation cardiovasculaire dans PROactive comparant la pioglitazone au placebo, et dans RECORD comparant la rosiglitazone avec la metformine ou un sulfamide hypoglycémiant. Les résultats concernent le nombre d'événements et le hazard ratio (HR) [avec l'intervalle de confiance à $95 \%$, IC $95 \%$ ]. Les données sont tirées de Dormandy et al. [8], Wilcox et al. [30] et Erdmann et al. [29] pour PROactive, et de Home et al. [36] et Komajda et al. [37] pour RECORD.

\begin{tabular}{|c|c|c|c|c|c|c|}
\hline \multirow[b]{2}{*}{ Critères de jugement } & \multicolumn{3}{|c|}{$\begin{array}{c}\text { PROactive } \\
\text { Inclusion : toute thérapie (sauf glitazone) } \\
\text { Suivi }=2,8 \text { années }\end{array}$} & \multicolumn{3}{|c|}{$\begin{array}{c}\text { RECORD } \\
\text { Inclusion : metformine (MET) ou } \\
\text { sulfamide hypoglycémiant (SU) } \\
\text { Suivi }=5,5 \text { années }\end{array}$} \\
\hline & $\begin{array}{l}\text { Pioglitazone } \\
\mathrm{n}=2605\end{array}$ & $\begin{array}{l}\text { Placebo } \\
n=2633\end{array}$ & $\begin{array}{c}\mathrm{HR} \\
{[\mathrm{IC} 95 \%]}\end{array}$ & $\begin{array}{c}\text { Rosiglitazone } \\
n=2220\end{array}$ & $\begin{array}{l}\text { SU ou MET } \\
n=2227\end{array}$ & $\begin{array}{c}\mathrm{HR} \\
\text { [IC } 95 \%]\end{array}$ \\
\hline Critère composite $\left(^{\star}\right)$ & 257 & 313 & $\begin{array}{c}0,82 \\
{[0,70-0,97]}\end{array}$ & 154 & 165 & $\begin{array}{c}0,93 \\
{[0,74-1,15]}\end{array}$ \\
\hline Décès de toutes causes & 177 & 186 & $\begin{array}{c}0,96 \\
{[0,78-1,18]}\end{array}$ & 136 & 157 & $\begin{array}{c}0,86 \\
{[0,68-1,08]}\end{array}$ \\
\hline Décès CV & 127 & 136 & $\begin{array}{c}0,94 \\
{[0,74-1,20]}\end{array}$ & 60 & 71 & $\begin{array}{c}0,84 \\
{[0,59-1,18]}\end{array}$ \\
\hline Infarctus du myocarde & 119 & 144 & $\begin{array}{c}0,83 \\
{[0,65-1,06]}\end{array}$ & 64 & 56 & $\begin{array}{c}1,14 \\
{[0,80-1,63]}\end{array}$ \\
\hline AVC & 86 & 107 & $\begin{array}{c}0,81 \\
{[0,61-1,07]}\end{array}$ & 46 & 63 & $\begin{array}{c}0,72 \\
{[0,49-1,06]}\end{array}$ \\
\hline $\begin{array}{l}\text { Insuffisance cardiaque } \\
\text { sévère }\end{array}$ & 149 & 108 & $\begin{array}{c}1,41 \\
{[1,10-1,80]}\end{array}$ & 61 & 29 & $\begin{array}{c}2,10 \\
{[1,35-3,27]}\end{array}$ \\
\hline $\begin{array}{l}\text { Insuffisance cardiaque } \\
\text { fatale }\end{array}$ & 25 & 22 & $\begin{array}{c}1,15 \\
{[0,65-2,03]} \\
\end{array}$ & 10 & 2 & $\begin{array}{c}5,0 \\
{[N D]} \\
\end{array}$ \\
\hline
\end{tabular}

CV : cardiovasculaire ; AVC : accident vasculaire cérébral ; ND : non disponible.

$\left(^{*}\right)$ Mortalité CV + infarctus du myocarde non mortels + AVC non mortels

courte durée, avec peu d'événements. En tenant compte de ces limitations, il existe une tendance, non significative, pour une réduction (approchant les $20 \%$ ) des IDM, une réduction de la mortalité totale (mais, étonnamment, pas de la mortalité $\mathrm{CV}$ ) et une augmentation, attendue, du risque d'insuffisance cardiaque (tableau II). Dans l'une de ces méta-analyses, le nombre d'événements $C V$, tous confondus, étaient significativement diminué dans le groupe pioglitazone par rapport au groupe contrôle (OR $=0,82$ [IC $95 \%: 0,72-0,94]$ ) [33]. Enfin, dans une comparaison des méta-analyses étudiant les effets de la pioglitazone et de la rosiglitazone, il a été montré des effets plus favorables au profit de la pioglitazone [35].

\section{Rosiglitazone}

\section{Essais cliniques contrôlés}

- RECORD est un essai multicentrique ouvert qui a recruté 4447 patients avec un DT2 imparfaitement contrôlé par une monothérapie par metformine ou sulfamide hypoglycémiant, chez lesquels a été ajoutée, de façon aléatoire, la 
rosiglitazone $(n=2220)$ en comparaison à une bithérapie metformine + sulfamide hypoglycémiant ( $n=2227)$ [36]. Après un suivi moyen de 5,5 années, le critère d'évaluation principal composite (décès $\mathrm{CV}$, ou hospitalisation pour une cause CV) est survenu de façon comparable dans les deux groupes (tableau IV). II en fut de même pour les décès $C V$, les IDM et les AVC. Comme attendu, les hospitalisations pour insuffisance cardiaque sont survenues plus fréquemment dans le groupe rosiglitazone (tableau IV) [36, 37].

- Dans l'étude BARI 2D, réalisée chez des patients DT2 avec une insuffisance coronaire, un traitement par rosiglitazone n'a pas été associé à une augmentation de la mortalité, ni des accidents $\mathrm{CV}$ ischémiques majeurs [38] : après ajustements pour les facteurs confondants, la mortalité de toutes causes (HR $=0,83$ [IC $95 \%$ : $0,58-1,18])$ et l'incidence des IDM (HR $=0,77$ [IC $95 \%: 0,54-1,10])$ ont été comparables sous rosiglitazone et sous comparateur, tandis que l'incidence d'un critère composite comprenant les décès, les IDM et les AVC ( $H R=0,72$ [IC $95 \%: 0,55-0,93]$ ) et celle des AVC ( $H R=0,36$ [IC $95 \%: 0,16-0,86])$ étaient même significativement réduites dans le groupe rosiglitazone [38].

\section{Études observationnelles de cohorte}

Les études observationnelles de cohorte sont relativement limitées et donnent des résultats discordants. En effet, une étude, réalisée en Israël, n'a pas montré d'augmentation significative du risque d'IDM, de syndrome coronaire aigu, de revascularisation coronaire ou de la mortalité totale sous rosiglitazone par comparaison à un traitement par metformine ; seul le risque d'insuffisance cardiaque était significativement accru, multiplié par 2 environ [39]. Lorsque I'on compare les deux thiazolidinediones, une étude anglaise [18] et une étude américaine [32] ont révélé un risque accru chez les patients traités par rosiglitazone en comparaison avec ceux traités par pioglitazone, alors qu'une étude réalisée à Taiwan, au contraire, a montré un pronostic meilleur sous rosiglitazone que sous pioglitazone [40].
Deux méta-analyses, l'une basée sur des études observationnelles [41], et l'autre sur des études rétrospectives de cohortes [42], ont confirmé une augmentation du risque d'IDM, d'insuffisance cardiaque et de mortalité avec la rosiglitazone par rapport à la pioglitazone. T3/Méta-analyses

- Les méta-analyses concernant la rosiglitazone ont rapporté des résultats contrastés. Nissen et Wolski ont publié, en 2007 , les résultats d'une première méta-analyse de 42 essais cliniques randomisés (40 essais de moindre envergure, plus ADOPT et DREAM) [9]. Ces essais rencontraient les critères suivants : durée de suivi d'au moins 24 semaines, l'existence d'un groupe recevant la rosiglitazone (4 ou $8 \mathrm{mg}$ ) et d'un groupe contrôle n'utilisant pas la rosiglitazone (placebo ou comparateur actif) et la disponibilité de données concernant les IDM et la mortalité CV. De façon inattendue, le risque s'est révélé plus élevé pour la survenue d'IDM $(\mathrm{OR}=1,43 ; p=0,03)$ et pour la mortalité $\mathrm{CV}(\mathrm{OR}=1,64 ; p=0,06)$ dans le groupe rosiglitazone par comparaison au groupe sans rosiglitazone (tableau II). Cette méta-analyse a jeté un pavé dans la mare puisqu'elle allait à l'encontre des espoirs fondés dans les glitazones. Comme on pouvait s'y attendre, elle a suscité, depuis lors, beaucoup de discussions et de controverses (voir plus loin, "Controverses au sujet du niveau de preuve $»$ ).

- Les mêmes auteurs ont confirmé les résultats initiaux dans une publication ultérieure, publiée en 2010, incluant les résultats du grand essai RECORD [43]. Le risque d'IDM demeurait accru, quoi que de façon moins marquée que dans leur méta-analyse initiale (OR $=1,28 ; p=0,04)$, tandis que le risque accru de mortalité $\mathrm{CV}$, suspecté initialement, a complètement disparu $(\mathrm{OR}=1,03 ; p=0,86$ ) (tableau II). Une autre méta-analyse des essais cliniques contrôlés n'a pas montré d'augmentation significative de l'incidence d'IDM, ni de la mortalité $\mathrm{CV}$, mais uniquement un accroissement du risque d'insuffisance cardiaque, en particulier chez les patients traités par insuline $(O R=2,2)$ (tableau II) [44]. Cependant, dans une autre méta-analyse des essais ayant inclus des patients DT2 traités par insuline, l'ajout de la rosiglitazone n'a pas augmenté le risque d'IDM ou de la mortalité par rapport à des patients traités par insuline seule, avec, par ailleurs, une amélioration métabolique (meilleur taux d'HbA $\mathrm{A}_{1 \mathrm{c}}$ avec une dose d'insuline moindre) [45]. Enfin, une méta-analyse, publiée récemment, de quatre grands essais contrôlés impliquant la rosiglitazone durant plus de 12 mois (alors que les analyses précédentes avaient inclus de nombreux essais de moins d'un an) n'a pas montré un sur-risque de la rosiglitazone par rapport au comparateur [46].

\section{Controverses au sujet du niveau de preuve}

\section{Metformine}

- La controverse concernant la metformine concerne essentiellement le niveau de preuve apporté par l'étude UKPDS. En effet, plusieurs critiques peuvent être émises à l'encontre du «bras metformine » de cette étude [13]: ainsi, le groupe metformine est limité et ne représente pas l'objectif principal de I'UKPDS, il n'y a pas eu de groupe placebo avec un suivi en double insu, les critères de jugement ont été modifié dans le décours de l'étude (dont la durée a été particulièrement longue), les traitements concomitants sont imparfaitement détaillés, et peut-être non répartis de façon équivalente [13]. - Les différentes méta-analyses disponibles donnent des résultats disparates, liés aux types d'essais retenus et au comparateur pris en compte, notamment [13, 25-27]. Quoi qu'il en soit, elles n'ont pas réussi à démontrer, de façon évidente, un effet protecteur de la metformine. II faut cependant mentionner que les essais inclus dans ces méta-analyses n'avaient pas été conçus pour étudier la problématique CV, et que le nombre d'événements était relativement faible dans l'immense majorité des essais disponibles. Le niveau de certitude de ces méta-analyses est donc assez faible.

- Au vu des effets pléiotropes de la metformine [47] et des résultats généralement positifs dérivés des études 
observationnelles - la plupart en comparaison avec des sulfamides hypoglycémiants, il faut le reconnaître [17-20] -, il serait temps de pouvoir disposer d'un grand essai clinique prospectif visant à démontrer l'effet protecteur de la metformine vis-à-vis des événements CV majeurs dans la population DT2, et plus particulièrement chez les patients à haut risque $\mathrm{CV}$ (comme ceux inclus dans les études de non-infériorité récemment publiées avec les inhibiteurs de la DPP-4 ou des inhibiteurs du cotransporteur sodium-glucose de type 2 [SGLT2]). Hélas, compte tenu du fait que la metformine n'est plus protégée par un brevet, une telle étude ne sera jamais mise sur pied par l'industrie pharmaceutique et devrait être financée par des organismes indépendants. De plus, il paraît très difficile de faire une étude versus un placebo, et le choix d'un comparateur actif s'avère, au stade actuel, très délicat parmi les différentes possibilités potentielles (un sulfamide hypoglycémiant ne paraît plus être un comparateur adéquat au stade actuel) [11]. II est à craindre que nous ne disposerons jamais d'une telle étude qui, pourtant, pourrait s'avérer décisive.

\section{Pioglitazone}

- La controverse à propos de la pioglitazone, concerne, d'une part, l'interprétation des résultats de l'étude PROactive et d'autre part, plus globalement, le rapport bénéfices/risques de la molécule.

- Les résultats de l'étude PROactive ont fait l'objet de beaucoup de débats, en particulier parce que le critère principal n'atteignait pas le seuil de la signification statistique, et que ce n'était que le dénommé " critère secondaire principal » qui remplissait cette condition [8]. Pour les experts des études cliniques, il s'agit donc d'une étude négative. Cependant, les cliniciens de terrain, non spécialistes en méthodologie des essais cliniques, ne manqueront pas d'être favorablement impressionnés par le fait que le critère secondaire principal est celui le plus largement utilisé dans les autres grandes études de préventions CV (celles avec les statines, par exemple), mais aussi que des analyses de sous-groupes (certes réalisées post hoc) ont montré une réduction significative d'événements CV majeurs [28] : récidives d'IDM chez les patients ayant déjà présenté une telle complication [29], et une diminution, encore plus marquée, des récidives des AVC [30].

- Par ailleurs, si la pioglitazone apporte une certaine protection $\mathrm{CV}$, il faut mettre celle-ci en balance avec d'autres manifestations indésirables attribués à cette glitazone, à savoir un risque accru d'insuffisance cardiaque, de fractures osseuses et, possiblement, quoique de plus en plus controversé au vu des dernières données disponibles, de cancers de vessie (suspicion qui a conduit au retrait de la pioglitazone en France) [48]. Dans ces conditions, le réel bénéfice clinique de la pioglitazone dépendra sans doute du profil individuel du patient. Néanmoins, lorsque l'on compare les résultats obtenus dans l'étude PROactive [8], pourtant décriés, avec ceux obtenus dans les trois grands essais de non-infériorité publiés récemment avec les inhibiteurs de la DPP-4 (SAVOR-TIMI 53, EXAMINE, et TECOS) [49], on ne peut manquer de penser que, finalement, les effets protecteurs $\mathrm{CV}$ obtenus avec la pioglitazone étaient loin d'être négligeables et devraient sans doute être reconsidérés. Un nouvel essai avec la pioglitazone serait, dans ce contexte, le bienvenu. Par ailleurs, au vu des résultats de l'étude EMPA-REG OUTCOME [50] avec l'empagliflozine, une combinaison inhibiteur des SGLT2 et pioglitazone pourrait s'avérer très attractive en termes de protection CV.

\section{Rosiglitazone}

- La controverse relative à la rosiglitazone a été amorcée, en 2007, avec la publication de la méta-analyse de Nissen et Wolski [9] et a abouti au retrait de la rosiglitazone dans de nombreux pays (mais pas aux États-Unis). Cependant, cette méta-analyse a été très critiquée et à plusieurs titres. Ainsi, aucun des essais inclus dans cette méta-analyse n'était conçu pour étudier la morbidité $\mathrm{CV}$, les protocoles étaient très disparates (posologie, durée du suivi, comparateur, profil de patients,...), les auteurs n'ont pas eu accès aux données source et ont dû se contenter des données publiées (non nécessairement validées sur le plan CV puisqu'il ne s'agissait pas là d'un critère de jugement principal) et le nombre total d'événements analysés, malgré le nombre important d'essais pris en compte, était relativement restreint [9]. Par ailleurs, certaines des études reprises dans la méta-analyse testaient la rosiglitazone hors indication officielle, dans des situations connues comme étant plus à risque, avec des patients diabétiques insulino-traités (indication non reconnue en Europe) ou même présentant à l'inclusion une décompensation cardiaque légère à modérée. Enfin, cette méta-analyse a exclu des études où il n'y avait eu aucun cas d'IDM ou de décès CV. Compte tenu des limites de cette méta-analyse, reconnues par les auteurs eux-mêmes [9], il convient sans doute de rester prudent avant de tirer quelque conclusion définitive, comme cela avait sans doute été fait un peu précipitamment, et les données actualisées de Nissen et Wolski, publiées en 2010, étaient déjà plus rassurantes [43]. Depuis, il apparaît de plus en plus que le risque attribué à la rosiglitazone a été surévalué [46] et la FDA, aux États-Unis, a progressivement allégé les mises en garde concernant l'utilisation de cette glitazone. Les méta-analyses ont certaines forces, mais aussi des limitations évidentes, en particulier lorsqu'elles prennent en compte des essais hétérogènes, non planifiés pour analyser le critère d'évaluation, à savoir les événements CV, en ce compris la mortalité CV et la mortalité totale. II n'empêche que les méta-analyses comparant la rosiglitazone et la pioglitazone plaident en faveur de la pioglitazone [35].

- L'histoire des antidiabétiques a toujours été faite de haut et de bas, que ce soit pour les sulfamides hypoglycémiants, les biguanides et les glitazones, comme discuté précédemment dans un éditorial à propos de l'émergence des médicaments à effet incrétine [51]. II n'est donc pas exclu que les glitazones, tombées en discrédit pour le moment (certainement en France), reprennent une certaine place dans la prise en charge de patients DT2 judicieusement sélectionnés, dans une approche personnalisée telle que celle recommandée dans les dernières recommandations [11]. 


\section{Conclusion}

- La sécurité CV de la metformine paraît établie au vu de l'ensemble des données disponibles. La démonstration de sa capacité à diminuer le risque vasculaire, considérée comme acquise depuis les résultats de l'étude UKPDS, mériterait confirmation dans une grande étude prospective contrôlée, spécifiquement dédiée à cette problématique, en particulier chez des patients à plus haut risque CV que ceux recrutés dans I'UKPDS. Ceci est d'autant plus important que la metformine est considérée comme le premier choix dans le traitement du DT2 et très largement utilisée en clinique, et que de nombreux patients DT2 sont, de fait, à haut risque CV. Dans les conditions de vie réelle, différentes études de cohorte ont rapportés un meilleur pronostic CV sous metformine que sous sulfamides hypoglycémiants, mais les données par rapport à d'autres comparateurs potentiels sont actuellement trop limitées pour pouvoir tirer une quelconque conclusion. Cette information serait essentielle pour pouvoir distinguer un effet protecteur de la metformine d'un éventuel effet délétère des sulfamides hypoglycémiants.

- Quant aux glitazones, leur profil d'activité sur l'insulinorésistance et les facteurs de risque CV associés apparait, a priori, encore plus séduisant que celui de la metformine. La pioglitazone se distingue de la rosiglitazone par un effet plus favorable sur le profil lipidique. Cependant, la capacité des thiazolidinediones à diminuer significativement le risque CV a été mise en doute après les résultats controversés de l'étude PROactive avec la pioglitazone, et les résultats globalement neutres de l'étude RECORD avec la rosiglitazone. En outre, la classe a été très négativement impactée par la méta-analyse, controversée par ailleurs, de Nissen et Wolski, et par certains bruits alarmistes concernant la pioglitazone. Ainsi, ces médicaments, qui agissent davantage sur l'action de l'insuline que sur sa sécrétion, n'ont pu émerger au sein de l'arsenal médicamenteux pour traiter le DT2 et sont, soit peu utilisés, soit même ont disparu du marché dans certains pays, dont la France. À ce stade, il est difficile de prédire quel sera le devenir des glitazones et $\mathrm{si}$, un jour, elles parviendront à démontrer de façon indiscutable un effet de protection CV.

Signification des acronymes des études citées

- ACCORD : Action to Control CardiOvascular Risk in Diabetes

- ADOPT : A Diabetes Outcome Progression Trial

- BARI 2D : Bypass Angioplasty Revascularization Investigation 2 Diabetes

- COSMIC : Comparative Outcomes Study of Metformin Intervention versus Conventional Approach Study

- DIGAMI 2 : Diabetes Mellitus InsulinGlucose Infusion in Acute Myocardial Infarction 2 Study

- DREAM : Diabetes Reduction Assessment with ramipril and rosiglitazone Medication

- EMPA-REG OUTCOME : Empagliflozin cardiovascular outcome events in type 2 diabetes mellitus patients

- EXAMINE : EXamination of cardiovascular outcomes: Alogliptin vs. standard of care in patients with type 2 diabetes mellitus and acute coronary syndrome

- PROactive : PROspective pioglitAzone Clinical Trial In macroVascular Events

- REACH : Reduction of Atherothrombosis for Continued Health

- RECORD : Rosiglitazone Evaluated for Cardiovascular Outcomes in oral agent combination therapy for type 2 Diabetes

- SAVOR-TIMI 53: Saxagliptin Assessment of Vascular Outcomes Recorded in patients with diabetes mellitus-Thrombolysis In Myocardial Infarction (TIMI) Group

- TECOS : Trial Evaluating Cardiovascular Outcomes with Sitagliptin

- UKPDS : United Kingdom Prospective Diabetes Study
NOTE : ce texte correspond à la présentation de l'auteur lors de la session «Rôle délétère de l'hyperglycémie: spécificités des différents traitements antidiabétiques " de la Journée Thématique 2015 de la Société francophone du diabète (SFD) "Certitudes et incertitudes liées à l'intérêt du contrôle glycémique ", Paris, 11 décembre 2015.

\section{Déclaration d'intérêt}

André J. Scheen déclare avoir reçu des honoraires, à titre personnel ou institutionnel, comme orateur, conseiller scientifique et/ou investigateur clinicien, de la part des firmes suivantes: AstraZeneca/Bristol-Myers Squibb, Boehringer Ingelheim, Eli Lilly, GlaxoSmithKline, Janssen, Merck Sharp \& Dohme, Novartis, Novo Nordisk, Sanofi, Takeda.

\section{Références}

[1] DeFronzo RA. Banting Lecture. From the triumvirate to the ominous octet: a new paradigm for the treatment of type 2 diabetes mellitus. Diabetes 2009;58:773-95.

[2] Reaven GM. Banting Lecture 1988. Role of insulin resistance in human disease. Diabetes 1988;37:1595-607.

[3] DeFronzo RA, Ferrannini E. Insulin resistance. A multifaceted syndrome responsible for NIDDM, obesity, hypertension, dyslipidemia, and atherosclerotic cardiovascular disease. Diabetes Care 1991;14:173-94.

[4] Scheen AJ, Charbonnel B. Effects of glucoselowering agents on vascular outcomes in type 2 diabetes: a critical reappraisal. Diabetes Metab 2014;40:176-85

[5] Effect of intensive blood-glucose control with metformin on complications in overweight patients with type 2 diabetes (UKPDS 34). UK Prospective Diabetes Study (UKPDS) Group. Lancet 1998;352:854-65 [Erratum in: Lancet 1998;352:1558].

[6] Foretz M, Guigas B, Bertrand L, et al. Metformin: from mechanisms of action to therapies. Cell Metab 2014;20:953-66.

[7] Yki-Järvinen $\mathrm{H}$. Thiazolidinediones. N Engl J Med 2004;351:1106-18.

[8] Dormandy JA, Charbonnel B, Eckland DJ, et al; PROactive investigators. Secondary prevention of macrovascular events in patients with type 2 diabetes in the PROactive Study (PROspective pioglitAzone Clinical Trial In macroVascular Events): a randomised controlled trial. Lancet 2005;366:1279-89.

[9] Nissen SE, Wolski K. Effect of rosiglitazone on the risk of myocardial infarction and death from cardiovascular causes. $\mathrm{N}$ Engl J Med 2007;356:2457-71 [Erratum in: N Engl J Med 2007;357:100]

[10] Lincoff AM, Tardif JC, Schwartz GG, et al AleCardio Investigators. Effect of aleglitazar on cardiovascular outcomes after acute coronary syndrome in patients with type 2 diabetes mellitus: the AleCardio randomized clinical trial. JAMA 2014;311:1515-25.

[11] Inzucchi SE, Bergenstal RM, Buse JB, et al. Management of hyperglycaemia in type 2 diabetes, 2015: a patient-centred approach. Update to a position statement of the American Diabetes Association and the European Association for the Study of Diabetes. Diabetologia 2015;58:429-42. [12] Holman RR, Paul SK, Bethel MA, et al. 10 -year follow-up of intensive glucose control in type 2 diabetes. N Engl J Med 2008;359:1577-89.

[13] Boussageon R, Supper I, Bejan-Angoulvant $\mathrm{T}$, et al. Reappraisal of metformin efficacy in the treatment of type 2 diabetes: a meta-analysis of randomised controlled trials. PLoS Med 2012;9:e1001204. 
[14] Cryer DR, Nicholas SP, Henry DH, et al. Comparative outcomes study of metformin intervention versus conventional approach: the COSMIC Approach Study. Diabetes Care 2005;28:539-43.

[15] Kooy A, de Jager J, Lehert P, et al. Long-term effects of metformin on metabolism and microvascular and macrovascular disease in patients with type 2 diabetes mellitus. Arch Intern Med 2009;169:616-25.

[16] Mellbin LG, Malmberg K, Norhammar A, et al; DIGAMI 2 Investigators. Prognostic implications of glucose-lowering treatment in patients with acute myocardial infarction and diabetes: experiences from an extended follow-up of the Diabetes Mellitus Insulin-Glucose Infusion in Acute Myocardial Infarction (DIGAMI) 2 Study. Diabetologia 2011;54:1308-17.

[17] Johnson JA, Majumdar SR, Simpson SH, Toth EL. Decreased mortality associated with the use of metformin compared with sulfonylurea monotherapy in type 2 diabetes. Diabetes Care 2002;25:2244-8.

[18] Tzoulaki I, Molokhia M, Curcin V, et al. Risk of cardiovascular disease and all cause mortality among patients with type 2 diabetes prescribed oral antidiabetes drugs: retrospective cohort study using UK general practice research database. BMJ 2009;339:b4731.

[19] Roumie CL, Hung AM, Greevy RA, et al. Comparative effectiveness of sulfonylurea and metformin monotherapy on cardiovascular events in type 2 diabetes mellitus: a cohort study. Ann Intern Med 2012;157:601-10.

[20] Morgan CL, Mukherjee J, Jenkins-Jones S, et al. Association between first-line monotherapy with sulphonylurea versus metformin and risk of all-cause mortality and cardiovascular events: a retrospective, observational study. Diabetes Obes Metab 2014;16:957-62.

[21] Chang $\mathrm{CH}$, Chang YC, Lin JW, et al. Cardiovascular risk associated with acarbose versus metformin as the first-line treatment in patients with type 2 diabetes: a nationwide cohort study. J Clin Endocrinol Metab 2015;100:1121-9. [22] Scheller NM, Mogensen UM, Andersson $\mathrm{C}$, et al. All-cause mortality and cardiovascular effects associated with the DPP-IV inhibitor sitagliptin compared with metformin, a retrospective cohort study on the Danish population. Diabetes Obes Metab 2014;16:231-6.

[23] Roussel R, Travert F, Pasquet B, et al: Reduction of Atherothrombosis for Continued Health (REACH) registry. Metformin use and mortality among patients with diabetes and atherothrombosis. Arch Intern Med 2010;170:1892-9,

[24] Scheen AJ, Paquot N. Metformin revisited: a critical review of the benefit-risk balance in at-risk patients with type 2 diabetes. Diabetes Metab 2013;39:179-90.

[25] Selvin E, Bolen S, Yeh $\mathrm{HC}$, et al. Cardiovascular outcomes in trials of oral diabetes medications: a systematic review. Arch Intern Med 2008;168:2070-80.

[26] Lamanna C, Monami M, Marchionni N, Mannucci E. Effect of metformin on cardiovascular events and mortality: a meta-analysis of randomized clinical trials. Diabetes Obes Metab 2011;13:221-8. [27] Hemmingsen B, Christensen LL, Wetterslev $\mathrm{J}$, et al. Comparison of metformin and insulin versus insulin alone for type 2 diabetes: systematic review of randomised clinical trials with metaanalyses and trial sequential analyses. BMJ 2012;344:e1771.

[28] Scheen AJ. Outcomes and lessons from the PROactive study. Diabetes Res Clin Pract 2012;98:175-86.

[29] Erdmann E, Dormandy JA, Charbonnel B, et al; PROactive Investigators. The effect of pioglitazone on recurrent myocardial infarction in 2,445 patients with type 2 diabetes and previous myocardial infarction: results from the PROactive (PROactive 05) Study. J Am Coll Cardiol 2007;49:1772-80.

[30] Wilcox R, Bousser MG, Betteridge DJ, et al; PROactive Investigators. Effects of pioglitazone in patients with type 2 diabetes with or without previous stroke: results from PROactive (PROspective pioglitAzone Clinical Trial In macroVascular Events 04). Stroke 2007;38:865-73.

[31] Seong JM, Choi NK, Shin JY, et al. Differential cardiovascular outcomes after dipeptidyl peptidase-4 inhibitor, sulfonylurea, and pioglitazone therapy, all in combination with metformin, for type 2 diabetes: a population-based cohort study. PLoS One 2015;10:e0124287.

[32] Ziyadeh N, McAfee AT, Koro C, et al. The thiazolidinediones rosiglitazone and pioglitazone and the risk of coronary heart disease: a retrospective cohort study using a US health insurance database. Clin Ther 2009;31:2665-77.

[33] Lincoff AM, Wolski K, Nicholls SJ, Nissen SE. Pioglitazone and risk of cardiovascular events in patients with type 2 diabetes mellitus: a meta-analysis of randomized trials. JAMA 2007;298:1180-8.

[34] Mannucci E, Monami M, Lamanna C, et al. Pioglitazone and cardiovascular risk. A comprehensive meta-analysis of randomized clinical trials. Diabetes Obes Metab 2008;10:1221-38.

[35] Schernthaner G, Chilton RJ. Cardiovascular risk and thiazolidinediones--what do metaanalyses really tell us? Diabetes Obes Metab 2010;12:1023-35.

[36] Home PD, Pocock SJ, Beck-Nielsen H, et al; RECORD Study Team. Rosiglitazone evaluated for cardiovascular outcomes in oral agent combination therapy for type 2 diabetes (RECORD): a multicentre, randomised, open-label trial. Lancet 2009;373:2125-35

[37] Komajda M, McMurray JJ, Beck-Nielsen H, et al. Heart failure events with rosiglitazone in type 2 diabetes: data from the RECORD clinical trial. Eur Heart J 2010;31:824-31.
[38] Bach RG, Brooks MM, Lombardero M, et al; BARI 2D Investigators. Rosiglitazone and outcomes for patients with diabetes mellitus and coronary artery disease in the Bypass Angioplasty Revascularization Investigation 2 Diabetes (BAR 2D) trial. Circulation 2013;128:785-94.

[39] Loebstein R, Dushinat M, Vesterman-Landes $J$, et al. Database evaluation of the effects of long-term rosiglitazone treatment on cardiovascular outcomes in patients with type 2 diabetes. J Clin Pharmacol 2011;51:173-80.

[40] Wang CC, Chen WL, Kao TW, et al. Incidence of cardiovascular events in which 2 thiazolidinediones are used as add-on treatments for type 2 diabetes mellitus in a Taiwanese population. Clin Ther 2011;33:1904-13 [Erratum in: Clin Ther 2012;34:508].

[41] Loke YK, Kwok CS, Singh S. Comparative cardiovascular effects of thiazolidinediones: systematic review and meta-analysis of observational studies. BMJ 2011;342:d1309.

[42] Chen X, Yang L, Zhai SD. Risk of cardiovascular disease and all-cause mortality among diabetic patients prescribed rosiglitazone or pioglitazone: a meta-analysis of retrospective cohort studies. Chin Med J (Engl) 2012;125:4301-6.

[43] Nissen SE, Wolski K. Rosiglitazone revisited: an updated meta-analysis of risk for myocardial infarction and cardiovascular mortality. Arch Intern Med 2010;170:1191-201.

[44] Mannucci E, Monami M, Di Bari M, et al. Cardiac safety profile of rosiglitazone: a comprehensive meta-analysis of randomized clinical trials. Int J Cardiol 2010;143:135-40.

[45] Lu Y, Ma D, Xu W, et al. Effect and cardiovascular safety of adding rosiglitazone to insulin therapy in type 2 diabetes: A meta-analysis. J Diabetes Investig 2015;6:78-86.

[46] Stone JC, Furuya-Kanamori L, Barendregt JJ, Doi SA. Was there really any evidence that rosiglitazone increased the risk of myocardial infarction or death from cardiovascular causes? Pharmacoepidemiol Drug Saf 2015;24:223-7.

[47] Bromage DI, Yellon DM. The pleiotropic effects of metformin: time for prospective studies. Cardiovasc Diabetol 2015;14:109.

[48] Lewis JD, Habel LA, Quesenberry CP, et al. Pioglitazone use and risk of bladder cancer and other common cancers in persons with diabetes. JAMA 2015;314:265-77.

[49] Scheen AJ. Gliptines, sécurité cardiovasculaire et insuffisance cardiaque : le point après l'étude TECOS. Rev Med Suisse 2015;11:1526-31.

[50] Zinman B, Wanner C, Lachin JM, et al; EMPAREG OUTCOME Investigators. Empagliflozin, cardiovascular outcomes, and mortality in type 2 diabetes. N Engl J Med 2015 Sep 17 [Epub ahead of print].

[51] Scheen AJ. Exenatide once weekly in type 2 diabetes. Lancet 2008;372:1197-8. 